\title{
Coherent microwave generation from a pseudospark cathode Cherenkov maser
}

\author{
H. Yin, W. He, G. R. M. Robb, A. D. R. Phelps, K. Ronald, and A. W. Cross \\ Department of Physics and Applied Physics, University of Strathclyde, Glasgow G4 ONG, United Kingdom
}

(Received 15 July 1998; published 17 February 1999)

\begin{abstract}
Experimental results are presented from the first free electron maser experiment to use a pseudospark electron source. A pulsed, $70-80 \mathrm{kV}, 10 \mathrm{~A}$ electron beam of normalized brightness $10^{11} \mathrm{~A} \mathrm{~m}^{-2} \mathrm{rad}^{-2}$ and current density $1 \mathrm{kA} \mathrm{cm}^{-2}$ from a pseudospark electron cathode was used to produce coherent microwave radiation via a Cherenkov interaction between the electron beam and the $\mathrm{TM}_{01}$ mode of a 60-cm-long alumina-lined waveguide. A gain of $29 \mathrm{~dB}$ was measured with an output power of $2 \mathrm{~kW}$ in the frequency range $25.5-28.6 \mathrm{GHz}$. The experimental results were found to be consistent with numerical simulations of Cherenkov amplification. [S1098-4402(99)00027-0]
\end{abstract}

PACS numbers: 52.75.Va, 41.60.Bq, 52.75.Ms, 52.80.-s

Free electron masers have been of general interest for over 20 years and discharges have been studied for much longer. The pseudospark (PS) discharge is, however, a more recently recognized class of discharge $[1,2]$ which has produced electron beams with the highest combined current density and brightness of any known type of electron source. This paper describes the first study of a PS-based electron beam source in a free electron maser. The main result of this work was the first production of coherent electromagnetic radiation by an electron beam from a PS discharge.

A pseudospark discharge is a low pressure, transient hollow cathode gas discharge which occurs in a special geometry and can use several kinds of gases, e.g., argon, nitrogen, and hydrogen. The background gas pressure is such that $p d$, the product of the gas pressure $p$ and the distance $d$ between the front faces of the cathode and anode, is on the left-hand side of the Paschen curve between the Paschen minimum and vacuum breakdown. The PS discharge offers the possibility of fast, high repetition rate, high power closing switches [3,4], and electron beam sources of high current density $\left(>10^{4} \mathrm{~A} \mathrm{~cm}^{-2}\right)$, high brightness (up to $10^{12} \mathrm{~A} \mathrm{~m}^{-2} \mathrm{rad}^{-2}$ ), small beam diameter $(<4 \mathrm{~mm})$, very low emittance (tens of $\mathrm{mm} \mathrm{mrad}$ ), and variable duration (tens of nanoseconds to hundreds of nanoseconds) [5,6]. It is therefore potentially very attractive as an electron beam source for high power sources of electromagnetic radiation, such as free electron lasers [7], cyclotron autoresonance masers [8], and Cherenkov masers [9-14]. In this paper we present results from the first free electron maser experiment to use a PS-produced beam. This experiment involved a Cherenkov maser amplifier driven by an electron beam of $10 \mathrm{~A}, 70-80 \mathrm{kV}$, diameter $3 \mathrm{~mm}$, and normalized brightness $10^{11} \mathrm{~A} \mathrm{~m}^{-2} \mathrm{rad}^{-2}$ from a PS cathode. A resonant Cherenkov interaction between this beam and the $\mathrm{TM}_{01}$ mode of an alumina-lined waveguide in the frequency range 25.5-28.6 GHz allowed amplification of low power broadband microwave radiation emitted by the PS discharge and resulted in a measured gain of $29 \mathrm{~dB}$.
A schematic outline of the PS-based Cherenkov maser amplifier is shown in Fig. 1. The main components of the experiment are the PS-based electron beam source, the magnetic field for beam transport, the Cherenkov interaction region, electrical/beam diagnostics, and the microwave launching/diagnostic system. A self-pinched electron beam was extracted from the anode of an 8-gap PS discharge chamber (Fig. 1). The moderately relativistic bright electron beam was transported through the interaction region by an 80 -cm-long, $\leq 0.3 \mathrm{~T}$ solenoidproduced magnetic field. The interaction region consisted of a section of cylindrical waveguide, $60 \mathrm{~cm}$ in length, $4.75 \mathrm{~mm}$ in radius, lined with a $1.75-\mathrm{mm}$-thick layer of dielectric (alumina, $\epsilon=9.5$ ). The microwave radiation generated in the interaction region was guided a further $60 \mathrm{~cm}$ in a cylindrical vacuum waveguide before being launched into free space by a $30 \mathrm{~cm}$ long conical horn. A well-calibrated microwave diagnostic system was situated $1 \mathrm{~m}$ from the antenna.

Fast electron beam diagnostics were designed, constructed, and calibrated having response times typically of a few nanoseconds, which permitted time-correlated study of the PS discharge process and the evolution of the electron pulse parameters. The discharge voltage was measured by an instantaneous capacitive voltage probe. The discharge current was monitored by an in-line current shunt. Two Rogowski coils were inserted into the system to measure the beam current entering and exiting the Cherenkov interaction region hence enabling the current transported through the system to be measured. A fieldfree collimator of cylindrical shape was fitted immediately after the anode to measure the beam brightness [15]. The 4D trace space volume occupied by the collimated beam electrons is equal to the $4 \mathrm{D}$ acceptance of the collimator when the electron beam is fully collimated. Simultaneous measurement of the electron beam energy and current after collimation therefore gives the normalized beam brightness.

The maser system was originally evacuated down to $2-$ 3 mtorr at the discharge chamber and $\sim 0.01$ mtorr at the 


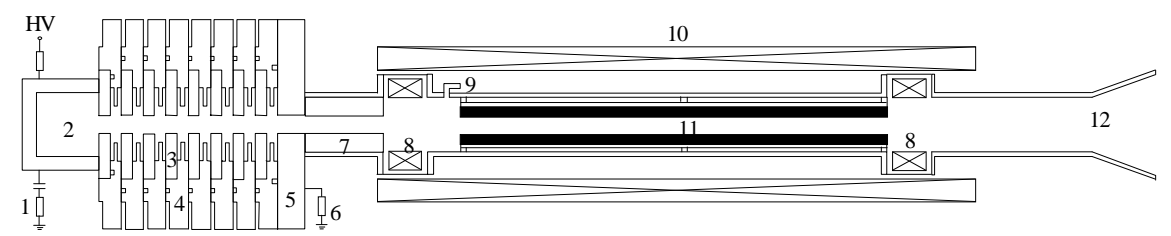

1 - Voltage probe 2 - Hollow cathode 3 - Intermediate electrode 4 - Insulator 5 - Anode 6 - CVR

7 - Collimator 8 - Rogowski coil 9 - Gas inlet 10 - Solenoid 11 - Dielectric liner 12 - Waveguide and horn

FIG. 1. Cherenkov maser experimental configuration.

pumping port. Argon gas was then fed into the system from the anode side. The pressure was increased at a slow rate of $\sim 1 \mathrm{mtorr} / \mathrm{s}$ until the desired gas pressure was reached and balanced by adjustment of a needle valve. The high voltage applied across the PS chamber was increased slowly until breakdown occurred. To achieve a self-pinched, high current electron beam it was essential to match the applied discharge voltage and chamber gas pressure so that the discharge operated in the PS discharge regime. Our experiments showed an empirical relation of $V_{b}=0.20 p^{-4.01} d^{-1.77}$, where $V_{b}$ is the breakdown voltage of the PS discharge in $\mathrm{kV}$, and $p$ and $d$ are the pressure in torr and the cathode-anode separation in centimeters, respectively.

The measured time-correlated PS discharge voltage and current displayed the three stages of a PS discharge: (1) Townsend discharge, (2) hollow cathode discharge, and (3) conductive PS discharge. The Townsend discharge in the PS gives rise to a plasma, i.e., a virtual anode, extending from the anode toward the cathode region. After entering the hollow cathode region, the plasma, with almost the full anode potential, forms a high field sheath surrounding the hollow cathode surface and gives rise to a very fast increase in the emission current. A high current axial electron beam, consisting mainly of electrons from the hollow cathode region behind the cathode hole, is formed during this hollow cathode discharge stage. Afterwards, the discharge transfers to the final conductive phase, in which the cold cathode surface facing the anode takes over the whole discharge current with a very high current and low acceleration potential until the gap is shorted [16-18].

The duration of the hollow cathode discharge of the PS cathode was adjustable between 10 and $50 \mathrm{~ns}$. The electron beam extracted in the hollow cathode stage had a current of 10-100 A with an acceleration potential close to the applied voltage. The electron beam from the conductive PS discharge had a current of 100-1000 A with a beam energy of a few hundred $\mathrm{eV}$. The normalized brightness of the electron beam was measured to be up to $10^{11}$ and $10^{12} \mathrm{~A} \mathrm{~m}^{-2} \mathrm{rad}^{-2}$ for the hollow cathode and conductive stages, respectively.

To obtain a stable high quality electron beam, essential for coherent microwave generation, care must be taken with appropriate gas feeding and pumping points and circuit parameters such as external capacitance value, etc. Also, in order to achieve the PS hollow cathode effects, our experiments showed that (i) the cathode hole diameter should be chosen to be in the range of the cathode thickness $(\sim 3 \mathrm{~mm})$ and (ii) the hollow cathode cavity depth should have a minimum value equivalent to the thickness of the cathode.

The presence of the dielectric in the waveguide reduces the phase velocity of the electromagnetic waves, allowing a resonant interaction to occur between a transverse magnetic (TM) or hybrid (HE) waveguide mode and the rectilinear electron beam. Coherence of the generated radiation arises due to bunching of electrons in phase with respect to the electromagnetic wave. For values of the beam and waveguide parameters relevant to this experiment, the force exerted on the electrons by the waveguide mode is dominated by the resonant spacecharge force, so the maser will operate in a Raman-type regime, with strongest amplification of the waveguide mode expected when it is resonant with the slow spacecharge wave of the beam, i.e., when the angular frequency $\omega$ and the axial wave number $k_{z}$ of the waveguide mode satisfy the relation

$$
\omega \approx k_{z} v_{z}-\frac{\omega_{p}}{\gamma},
$$

where $v_{z}$ is the axial electron velocity, $\gamma=1+$ $|e V| / m_{0} c^{2}$ is the relativistic factor, $\omega_{p}=\sqrt{e^{2} n_{e} / \epsilon_{0} \gamma m_{0}}$ is the plasma frequency, $n_{e}$ is the electron density, $V$ is the beam voltage, and $e$ and $m_{0}$ are the electronic charge and rest mass, respectively. $\omega$ and $k_{z}$ must also, of course, satisfy the characteristic equation for a dielectric-lined cylindrical waveguide, which, for a $\mathrm{TM}_{0 n}$ mode, is

$$
\frac{\epsilon \alpha_{2} I_{0}\left(\alpha_{2} a\right)}{\gamma_{1} I_{1}\left(\alpha_{2} a\right)}=\frac{J_{0}\left(\gamma_{1} a\right) Y_{0}\left(\gamma_{1} b\right)-J_{0}\left(\gamma_{1} b\right) Y_{0}\left(\gamma_{1} a\right)}{J_{1}\left(\gamma_{1} a\right) Y_{0}\left(\gamma_{1} b\right)-J_{0}\left(\gamma_{1} b\right) Y_{1}\left(\gamma_{1} a\right)},
$$

where $\gamma_{1}^{2}=\epsilon \omega^{2} / c^{2}-k_{z}^{2}, \alpha_{2}^{2}=k_{z}^{2}-\omega^{2} / c^{2}, a$ is the inner radius of the dielectric, $b$ is the outer radius of the dielectric, $\epsilon$ is the dielectric constant of the liner, and $J_{m}(x), Y_{m}(x)$, and $I_{m}(x)$ are $m$ th-order Bessel functions of the first kind, Bessel functions of the second kind, and modified Bessel functions of the first kind, respectively. Figure 2 shows the slow space-charge mode of the 


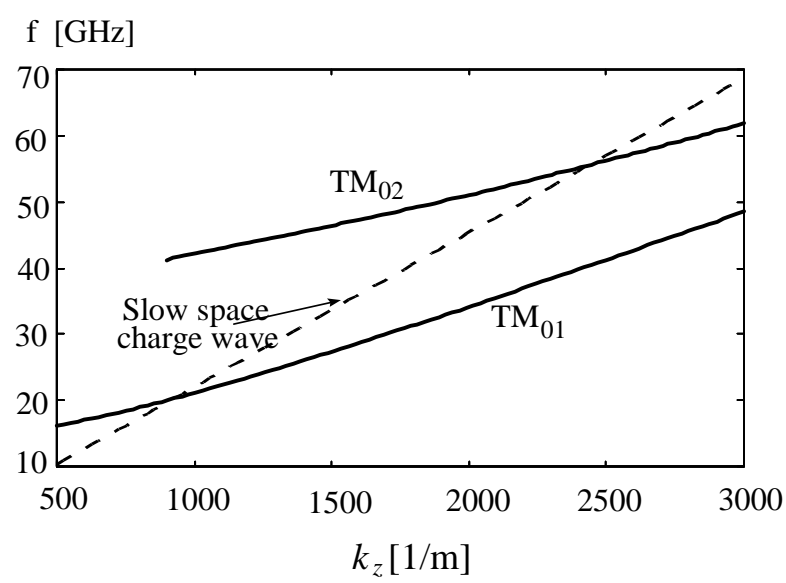

FIG. 2. Dispersion diagram showing the waveguide modes and slow space-charge mode of the electron beam $(75 \mathrm{kV}, 10 \mathrm{~A})$.

electron beam and the $\mathrm{TM}_{01}$ and $\mathrm{TM}_{02}$ waveguide modes for this Cherenkov maser experiment. From this diagram, a resonant interaction would be expected to occur around $21 \mathrm{GHz}$ with the $\mathrm{TM}_{01}$ mode and around $55 \mathrm{GHz}$ with the $\mathrm{TM}_{02}$ mode.

The temporal profile of the microwave radiation from the PS-based Cherenkov maser amplifier was obtained by monitoring the output of a rectifying crystal detector with an oscilloscope, time correlated with the electron-beam current and voltage profiles (Fig. 3). The microwave pulse was found to coincide with the hollow cathode discharge stage of the PS discharge allowing for the propagation time of the wave to the detector $(\approx 14 \mathrm{~ns})$ and the time delay due to the different cable lengths used for beam and microwave diagnostics $(\approx 5 \mathrm{~ns})$. It was observed that the amplitude of the output microwave pulse was independent of the guide magnetic field over the range 0.13 to $0.26 \mathrm{~T}$ and that removal of the dielectric liner resulted in the loss of microwave amplification.

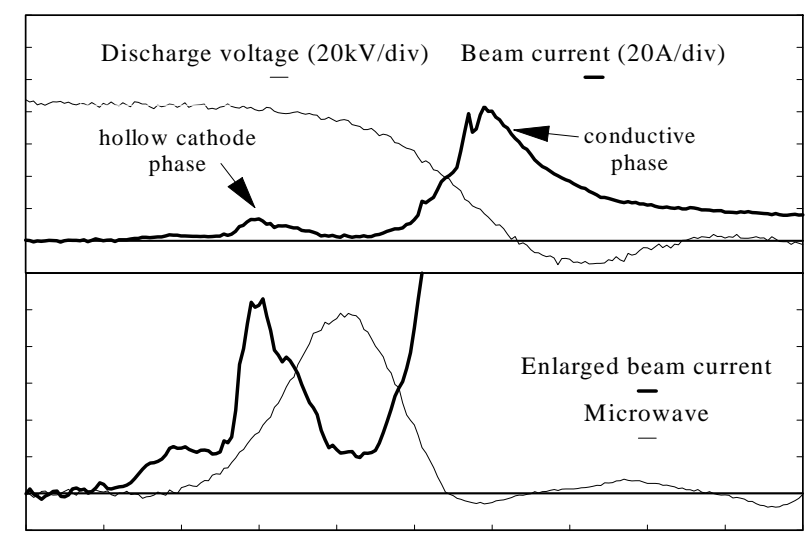

Time (20ns/div)

FIG. 3. Typical waveforms of the discharge voltage, the beam current, and the microwave pulse.
The frequency content of the microwave signal with and without Cherenkov amplification for an applied discharge voltage of $75 \mathrm{kV}$ was analyzed using a series of in-waveguide cutoff filters. By repetitively pulsing the maser, an averaged microwave output spectrum as a function of time was built up by normalizing the filtered signal to an unfiltered reference signal. The results showed that the signal in the absence of the dielectric, i.e., PS discharge radiation [19], had a wide frequency distribution but below $W$-band $(62.5 \mathrm{GHz})$ cutoff frequency. In the presence of the dielectric, the microwave output had typically two frequency components: one was between 25.5 and $28.6 \mathrm{GHz}$, and the other between 28.6 and $41.8 \mathrm{GHz}$. By comparing the time evolution of the beam voltage with the averaged microwave output spectrum, it was found that the low frequency microwave signal corresponded to a 70-80 keV beam energy from the hollow cathode discharge regime and the higher frequency microwave signal to a lower energy beam. This increase in frequency with decreasing beam energy is consistent with a Cherenkov interaction mechanism.

The observed frequency was found to be $20 \%$ higher than that predicted by the resonance condition for a 70$80 \mathrm{keV}$ beam energy. This discrepancy is probably due to charging of the dielectric liner (see, e.g., Fig. 8 of [14]). A relative spectral energy distribution over the frequency ranges was obtained and approximately 65\% of the radiation was found to lie in the $25-28.6 \mathrm{GHz}$ frequency band.

To verify the mode of operation as $\mathrm{TM}_{01}$, the farfield output radiation pattern from a conical antenna horn with an aperture diameter of $60 \mathrm{~mm}$ was measured. The output microwave pattern associated with the azimuthal $E$-field component was measured to be independent of the presence of the dielectric and close to zero, confirming the operation of a TM mode. Figure 4 shows the measured pattern associated with the radial $E$-field component of the radiation with a standard error of $4 \%$ and a systematic

Normalized microwave output ( $\mathrm{E}_{\mathrm{r}}$ component)

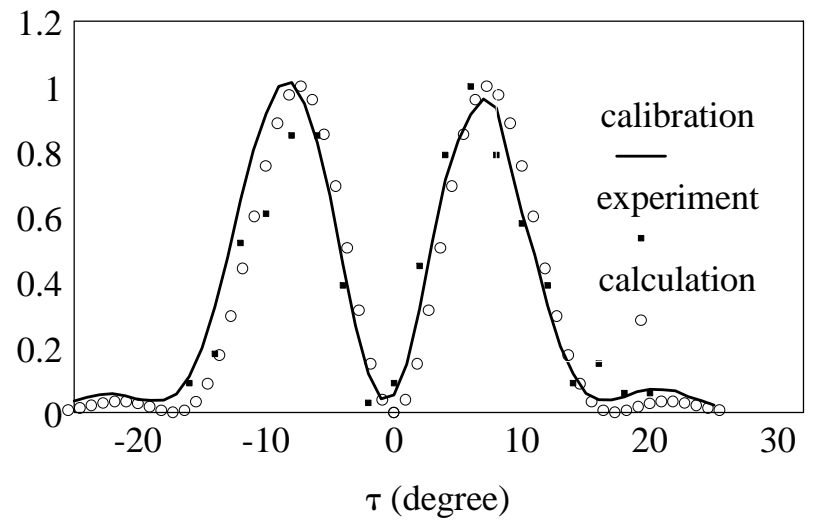

FIG. 4. Far-field mode pattern scan of the radial component $E_{r}$ compared with a calculation and bench calibration. 


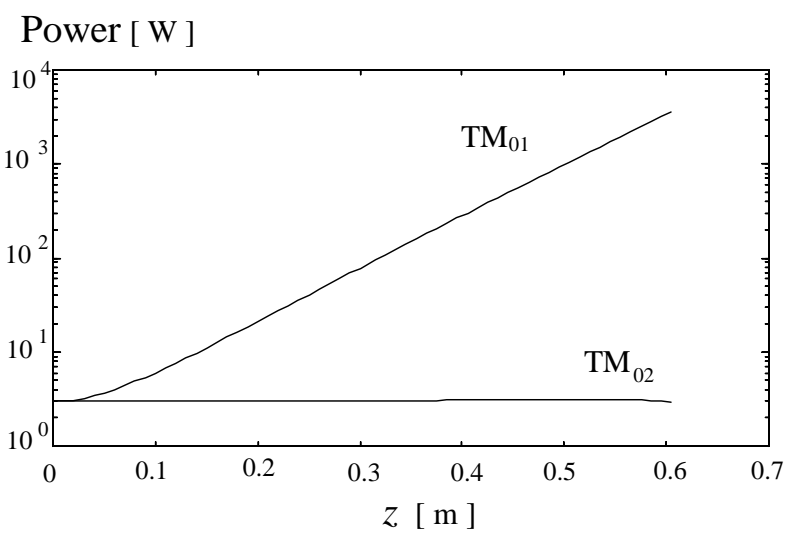

FIG. 5. Calculated output power of $\mathrm{TM}_{01}$ and $\mathrm{TM}_{01}$ modes as a function of $z$.

error in the radial angle measurement of $<2^{\circ}$. The measured pattern was in good agreement with the results from bench experiments in which a $27 \mathrm{GHz} \mathrm{TM}_{01}$ microwave signal was launched using the same horn. Fraunhofer diffraction theory predicts a maximum at $7.5^{\circ}$, which is in close agreement with both the Cherenkov maser experiment and bench measurements.

In order to measure the absolute power of the microwave pulse from the Cherenkov maser, the microwave energy received by a $15 \mathrm{~dB}$ gain rectangular receiving horn with an effective area of $3.5 \mathrm{~cm}^{2}$ (for the microwave frequency of $25.5 \mathrm{GHz}$ ) was fed to a well-calibrated crystal detector. The power density $1 \mathrm{~m}$ away at its maximum position was measured to be $1.6 \pm 0.2 \mathrm{~W} \mathrm{~cm}^{-2}$. Therefore the maximum total power from the Cherenkov device was calculated to be $2.0 \pm 0.2 \mathrm{~kW}$ (without considering any losses within the detecting system) by integrating the measured normalized mode pattern. After similar treatment, the microwave signal without maser amplification was found to be $2.7 \mathrm{~W}$ in the frequency range between 25.5 and $28.6 \mathrm{GHz}$. This corresponded to a gain of $29 \pm 3 \mathrm{db}$.

To complement the experimental investigations of the Cherenkov maser, a three-dimensional numerical simulation code was developed. The method used in this code is similar to that used in the Cherenkov maser simulation code of Freund [20]. The code integrates the Lorentz equations of motion for the electrons self-consistently with Maxwell's wave equation for a single $\mathrm{TM}_{0 n}$ waveguide mode in the steady-state limit where the relative slippage of the radiation with respect to the electron beam is neglected. It includes the effect of resonant spacecharge forces and electron energy spread. Figure 5 shows a graph of the predicted output power from the Cherenkov maser as a function of interaction length for the $\mathrm{TM}_{01}$ and $\mathrm{TM}_{02}$ modes when the beam energy is $75 \mathrm{keV}$ and the current is $10 \mathrm{~A}$. Only these two modes lie within the frequency range of the radiation emitted by the PS discharge. The microwave power at $z=0$ was assumed to be $3 \mathrm{~W}$ for both modes. The electron beam was assumed to be perfectly collimated, with an axial velocity spread $\left(\Delta v_{z} / v_{z}\right)$ of $3 \%$ and a radius of $1.5 \mathrm{~mm}$. It can be seen from Fig 5 that the $\mathrm{TM}_{01}$ mode at $\sim 21 \mathrm{GHz}$ is amplified strongly, attaining a power of $\sim 3.4 \mathrm{~kW}$ at $z=$ $60 \mathrm{~cm}$, whereas the power in the $\mathrm{TM}_{02}$ mode at $\sim 55 \mathrm{GHz}$ remains around its initial level. These simulations support the interpretation of the experimental results as microwave amplification via a Cherenkov interaction between the high-quality electron beam and the $\mathrm{TM}_{01}$ mode of the dielectric-lined waveguide.

In conclusion, we have presented the first coherent electromagnetic radiation generation by a free electron maser using an electron beam from a pseudospark discharge. The microwave radiation was generated by Cherenkov amplification of the broadband radiation emitted by the PS discharge. The frequency of the microwaves produced by the Cherenkov maser was measured to be mainly in the range $25.5-28.6 \mathrm{GHz}$, and the dominant mode was identified as being $\mathrm{TM}_{01}$. A peak power of $2.0 \pm 0.2 \mathrm{~kW}$ and a gain of $29 \pm 3 \mathrm{~dB}$ were achieved. The experimental results were consistent with numerical simulations. This high brightness PS-produced beam can, in principle, be used to drive many types of free electron masers with high efficiencies.

\section{ACKNOWLEDGMENTS}

One of us (H.Y.) would like to thank the Committee of Vice Chancellors and Principals of the Universities of the United Kingdom and Strathclyde University for support. Assistance from P. Aitken and C. Whyte is also gratefully acknowledged.

[1] M. A. Gundersen and G. Schaefer, in Physics and Applications of Pseudosparks, NATO ASI Series B, Vol. 219 (Plenum, New York, 1990).

[2] K. Frank and J. Christiansen, IEEE Trans. Plasma Sci. 17, 748 (1989).

[3] G. F. Kirkman and M. A. Gundersen, Appl. Phys. Lett. 49, 494 (1986).

[4] H. Riege and E. Boggasch, IEEE Trans. Plasma Sci. 17, 775 (1989).

[5] E. Boggasch and M. J. Rhee, Appl. Phys. Lett. 56, 18 (1990).

[6] W. Benker et al., IEEE Trans. Plasma Sci. 17, 754 (1989).

[7] T.C. Marshall, Free Electron Lasers (Macmillan, New York, 1985).

[8] S. J. Cooke, A. W. Cross, W. He, and A. D. R. Phelps, Phys. Rev. Lett. 77, 4836 (1996).

[9] J.E. Walsh, T.C. Marshall, and S. Schleshinger, Phys. Fluids 20, 709 (1977).

[10] K. L. Felch, K. O. Busby, R. W. Layman, D. Kapilow, and J. H. Walsh, Appl. Phys. Lett. 38, 601 (1981).

[11] S. Von Laven, J. Branscum, J. Golub, R. Layman, and J. Walsh, Appl. Phys. Lett. 41, 408 (1982). 
[12] W. Peter, E. Garate, W. Main, and A. Fisher, Phys. Rev. Lett. 65, 2989 (1990).

[13] W. B. Case et al., J. Appl. Phys. 55, 2651 (1984).

[14] E. Garate et al., J. Appl. Phys. 58, 627 (1985).

[15] H. Yin, Ph.D. thesis, Strathclyde University, 1998.

[16] P. Choi, R. Aliaga, and B. Blottiere, Appl. Phys. Lett. 63, 2750 (1993).

[17] M.P. Alberta, J. Derouard, L. C. Pitchford, N. Ouadoudi, and J. P. Boeuf, Phys. Rev. E 50, 2239 (1994).

[18] A. Anders, S. Anders, and M. A. Gundersen, Phys. Rev. Lett. 71, 364 (1993).

[19] R. Liou, H. Figueroa, A. H. McCurdy, G. KirkmanAmemiya, R. J. Temkin, H. Fetterman, and M. A. Gunderson, Appl. Phys. Lett. 61, 2779 (1992).

[20] H. P. Freund, Phys. Rev. Lett. 65, 2989 (1990). 